\title{
Interpretation Misunderstandings about Elementary Quantum Mechanics
}

\author{
Confusiones de interpretación en Mecánica Cuántica elemental \\ Federico G. Lopez Armengol ${ }^{\dagger}$ \\ Gustavo E. Romero
}

\begin{abstract}
Quantum Mechanics is a fundamental physical theory about atomic-scale processes. It was built between 1920 and 1940 by the most distinguished physicists of that time. The accordance between the predictions of the theory and experimental results is remarkable. The physical interpretation of its mathematical constructs, however, raised unprecedented controversies. Ontological, semantic, and epistemic vagueness abound in the orthodox interpretations and have resulted in serious misunderstandings that are often repeated in textbooks and elsewhere. In this work, we identify, criticize, and clarify the most spread ones.
\end{abstract}

Keywords: elementary quantum mechanics - interpretation - misunderstandings - Bunge

\section{Resumen}

La Mecánica Cuántica es una teoría de física fundamental que modela procesos a escalas atómicas. La teoría fue formulada entre los años 1920 y 1940. El acuerdo entre las predicciones obtenidas a partir de su formalismo matemático y los resultados experimentales es notable. Sin embargo, las interpretaciones físicas de los constructos de la teoría originaron controversias sin precedentes en la historia de la Física. Las imprecisiones ontológicas, semánticas y epistémicas de las distintas interpretaciones han ocasionado que se repitan y propaguen graves malentendidos que obstaculizan la investigación básica. En este trabajo identificaremos, criticaremos y aclararemos algunas de estas confusiones, con énfasis en las más básicas y difundidas.

Palabras claves: mecánica cuántica elemental - interpretación - confusiones - Bunge

\footnotetext{
"Recibido: 16 de Febrero de 2016. Aceptado con revisiones: 19 de Septiembre de 2016.

† Instituto Argentino de Radioastronomía CCT La Plata (CONICET), C.C.5 1894 Villa Elisa, Buenos Aires, Argentina. Para contactar al autor, por favor, escribir a: flopezar@iar-conicet.gov.ar

‡ Facultad de Ciencias Astronómicas y Geofísicas, Universidad Nacional de La Plata, Paseo del Bosque s/n, 1900 La Plata, Buenos Aires, Argentina. Para contactar al autor, por favor, escribir a: romero@iar-conicet.gov.ar. Metatheoria 7(2)(2017): 55-60. ISSN 1853-2322.

(c) Editorial de la Universidad Nacional de Tres de Febrero. Publicado en la República Argentina.
} 


\section{Introduction}

Modeling atomic-scale physical systems with classical laws yields inaccurate results. This fact was noticed in the first half of the XX century with phenomena such as black body radiation, the photoelectric effect, Stern-Gerlach deflections, and the Comptom effect. A battery of novel experiments based on these and other phenomena, along with remarkable theoretical work, led to the formulation of Quantum Mechanics (QM). The list of physicists that contributed to the new theory is long and includes illustrious names: Max Planck, Niels Bohr, Werner Heisenberg, Albert Einstein, Erwin Schrödinger, Max Born, John von Neumann, Wolfgang Pauli, David Hilbert, Paul Dirac, and Pascual Jordan among many others.

The predictions made with the theory were confirmed by several experimental measurements and the mathematical formalism was rapidly accepted by most physicist of the time. Nevertheless, the interpretation of the new theoretical constructs was far from clear and raised serious controversies. From this intellectual conflict, numerous interpretations of QM emerged. To name just a few: the Copenhagen interpretation, the de Broglie-Bohm theory, interpretations based on Quantum Logic, Time-Symmetric theories, the Many-Worlds interpretation, statistical interpretations, and realistic ones (for a review of QM interpretations and their historical context, see Jammer 1974)

The most accepted and spread interpretation of QM is the Copenhagen interpretation, proposed by Niels Bohr and Werner Heisenberg in 1927. The popularity of this view resides on its practical usefulness. The Copenhagen interpretation is, however, implicitly influenced by subjective and pragmatic philosophy.

In this work, we briefly describe a realistic interpretation of QM. Then, we discuss some widely used statements that are usually taught in QM elementary courses and sometimes even invoked in academic discussions. We hope to clarify some misunderstandings about interpretation issues related to the deterministic character of the theory, the so-called uncertainty principles, the wave function "collapse", the Einstein-Podolsky-Rosen (EPR) paradox, and the related ontology. The main reference of the article is Bunge (1967), but the reader might also benefit from Bunge (1973, 2010), and the discussion in Bunge (1983).

\section{Realistic approach}

We present a short description of the formalism of QM from a realistic perspective. We do not intend to be exhaustive, but rather to focus on those postulates that are key for understanding the theory. We include some equations using bra-ket notation, but mostly we are interested on semantic issues.

The referents of QM are particular physical systems called quantum systems. This statement may sound trivial, but is crucial for properly understanding the theory. We discuss it in Section 3.5.

The states of a quantum system are represented by non-unique unit vectors $|\psi\rangle$ in some Hilbert space $\mathcal{H}$, known as the state space, with a defined inner product. The state space $\mathcal{H}$ is isomorphic to $L_{2}\left(\mathbb{R}^{3 N}\right)$, the set of square integrable functions on the configuration space of the system. For this reason, it is usual to associate square integrable normalized functions on configuration space $\psi(\bar{p})$ with quantum states. The latter function is called wave function and is a fundamental tool for calculating the properties of the quantum systems.

Then, unlike classical theories, quantum states are represented by vectors in a space where a summation operation is defined. This fact and the linearity of the dynamic equations of the theory imply that the Principle of Superposition holds at the level of states. Consider, for instance, the wave functions $\left|\psi_{+}\right\rangle,\left|\psi_{-}\right\rangle$that represent the states of an electron with its spin up and down, respectively. Then, the state:

$$
|\psi\rangle=\frac{3}{5}\left|\psi_{+}\right\rangle+\frac{4}{5}\left|\psi_{-}\right\rangle
$$


represents a plausible quantum system that is a superposition of the spin-up and spin-down electron. For empirical confirmation of such counterintuitive feature of QM, called quantum entanglement, see Schlosshauer (2007), p. 21.

The values of the properties of the quantum systems can be calculated with self-adjoint operators $\hat{\mathcal{A}}(t): \mathcal{H} \rightarrow \mathcal{H}$, acting on the corresponding states. But, unlike classical systems, quantum systems may not have precise values for its properties. Instead, we can calculate the average $\langle\hat{\mathcal{A}}\rangle$ of a certain property by:

$$
\langle\hat{\mathcal{A}}\rangle=\langle\psi|\hat{\mathcal{A}}| \psi\rangle \text {. }
$$

The spread $\Delta_{\psi} \mathcal{A}$ of the average is:

$$
\Delta_{\psi} \hat{\mathcal{A}}^{2}=\langle\hat{\mathcal{A}}\rangle^{2}-\left\langle\hat{\mathcal{A}}^{2}\right\rangle
$$

If the spread $\Delta_{\psi} \hat{\mathcal{A}}$ of a certain property of a quantum state $\left|\psi_{k}\right\rangle$ is null, then the property takes a precise value $\lambda_{k}$. The corresponding state $\left|\psi_{k}\right\rangle$ is called eigenstate of the operator $\hat{\mathcal{A}}, \lambda_{k}$ its eigenvalue, and they satisfy:

$$
\hat{\mathcal{A}}\left|\psi_{k}\right\rangle=\lambda_{k}\left|\psi_{k}\right\rangle
$$

Under certain conditions, the values $\lambda_{k}$ may constitute a countable set, i.e. may be quantized. This is another peculiar and contrastable feature of QM. The name of the theory comes from this feature.

Because of the Superposition Principle, quantum states are not exclusive. For example, given an eigenstate $\left|\psi_{k}\right\rangle$ of certain self-adjoint operator $\hat{\mathcal{A}}(t)$, the propensity $p_{k}$ of any quantum state $|\psi\rangle$ to take the value $\lambda_{k}$ is measured by a probability:

$$
p_{k}=\left|\left\langle\psi \mid \psi_{k}\right\rangle\right|^{2}
$$

where $0<p_{k}<1$ (see Popper 1959).

Finally, QM has an evolution equation that describes how properties change with time. The equation reads:

$$
\frac{d \hat{\mathcal{A}}}{d t}=\frac{i}{\hbar}(\widehat{\mathcal{H}} \hat{\mathcal{A}}-\hat{\mathcal{A}} \widehat{\mathcal{H}})+\frac{\partial \hat{\mathcal{A}}}{\partial t},
$$

where $\widehat{\mathcal{H}}$ denotes a particular operator called Hamiltonian of the system.

We have specified the referents of QM, how to calculate their properties, and their dynamical equation. This short explanation suffices to clarify some usual misconceptions about quantum systems. For a detailed realistic formulation of the formalism see Perez Bergliaffa et al. $(1993,1996)$.

\section{Interpretation misunderstandings}

\subsection{Determinism}

It is usually argued that: "QM is not deterministic as Classical Mechanics because it cannot predict precisely the properties of its referents". The latter argument is misleading and needs clarification. This subsection is based mostly on Earman (1986).

Determinism is a polysemic word. We find numerous definitions in the literature but none of them refers to the accuracy of predictions. Instead, they refer to dynamical properties of the theory.

QM dynamic equation is invariant under time reversal if we accept that time reversal operation is given by $[\psi(\bar{p})]^{R} \rightarrow \psi^{*}(\bar{p})$ and $\hat{\mathcal{A}}(-t) \rightarrow \hat{\mathcal{A}}^{*}(t)$, where * denotes complex conjugation. On the other hand, QM evolution equation admits unique solutions under precise initial conditions. Classical theories do not possess this attribute because of possible disturbances coming from spatial infinity with unbounded velocity. QM forbids the invasion from spatial infinity because, in order to keep the wave 
function normalized, $\lim _{x \rightarrow \pm \infty} \psi(\bar{x})=0$. Moreover, the normalization of the wave function entails stability under variations of the initial conditions. This is not the general case in Classical Mechanics.

In summary, determinism does not refer to predictability, but to features of the time evolution. In this sense, we find that QM is even more deterministic than Classical Mechanics.

It is true that QM does not predict precise values for the properties of the system. However, that is not a problem of QM dynamics, but of its ontology. Quantum systems, as characterized by the standard formalism, do not have precise values for their properties; that's why we cannot predict them.

\subsection{Uncertainty relations}

Under the misleading name of uncertainty principles, it is stated that: "Given a quantum system, we cannot measure simultaneously and with arbitrary precision the values of properties whose associated operators do not commute". ${ }^{1}$ The main reference for this subsection is Bunge (1967).

First, the mentioned statement is based on a theorem; it is not a principle. The theorem reads: "Let $\hat{\mathcal{A}}, \widehat{\mathcal{B}}, \hat{\mathcal{C}}$ be self-adjoint operators such that $[\hat{\mathcal{A}}, \widehat{\mathcal{B}}]=i \hat{\mathcal{C}}$, then $\Delta_{\psi} \hat{\mathcal{A}} \Delta_{\psi} \widehat{\mathcal{B}} \geq\left|\langle\mathcal{C}\rangle_{\psi}\right| / 2$, for any quantum state $\psi "$.

The theorem does not make any reference to measurements processes, neither to unavoidable disturbances caused by our interaction with the system, nor to our incapability to measure precise quantum properties. It makes reference to the averaged properties of the quantum systems. In short, epistemic uncertainty is distinct from ontological variance of properties.

Furthermore, it is usually argued that time and energy satisfy an uncertainty relation of the form: $\Delta_{\psi} E \Delta_{\psi} t \geq \hbar / 2$. This relation is alien to QM formalism simply because the parameter time does not have an associated operator and, therefore, does not apply to the mentioned theorem (see Bunge, 1970). The correct time dependent uncertainty relation between the energy $E$ and a self-adjoint operator $\hat{\mathcal{A}}$ is (Messiah 1981):

$$
\frac{\Delta_{\psi} \hat{\mathcal{A}}}{\left|d\langle\hat{\mathcal{A}}\rangle_{\psi}\right| / d t} \Delta_{\psi} E \geq \frac{\hbar}{2}
$$

\subsection{The wave function collapse}

The most controversial postulate of the Copenhagen interpretation is the Projection Postulate: "If the measurement of a property $\hat{\mathcal{A}}$ of a quantum system $|\psi\rangle$ gives the result $\lambda_{k}$, where $\lambda_{k}$ is an eigenvalue of $\hat{\mathcal{A}}$, the quantum state $|\psi\rangle$ immediately changes to the corresponding eigenstate $\left.\left|\psi_{k}\right\rangle\right\rangle$.

First, we remark that QM is a fundamental physical theory that models the behavior of its referents, i.e. quantum systems. There is no place in its formalism for observers, human measurements, or classical entities. We admit that understanding the quantum-classical interaction is essential, and the Projection Postulate contributes on that sense, but it does not belong to QM proper. It should be a consequence of a theory, yet inexistent, that formally models quantum systems, classical apparatus, and their interactions, i.e. a quantum theory of measurement.

The Projection Postulate is a valuable pragmatic statement, but should be replaced by a formal model of the quantum-classical interaction. Moreover, strictly speaking mathematical objects such as functions in a Hilbert space cannot collapse. Only the physical systems can change their state or "collapse". The representation of physical collapse should not be confused with the collapse of mathematical objects.

\subsection{The Einstein-Podolsky-Rosen paradox}

Einstein, Podolsky and Rosen (1935) argued that formalism of QM was either incomplete, or unrealistic. The argument is based on a thought experiment about an entangled quantum state, whose components are arbitrarily distant from each other. The apparent paradox resides on instantaneous

\footnotetext{
${ }^{1}$ Two operators $\hat{\mathcal{A}}, \widehat{\mathcal{B}}$ do not commute if, and only if, $i \widehat{\mathcal{C}}=[\hat{\mathcal{A}}, \widehat{\mathcal{B}}]=\hat{\mathcal{A}} \widehat{\mathcal{B}}-\widehat{\mathcal{B}} \hat{\mathcal{A}} \neq 0$.
} 
effects over one of the components, produced by the local interaction of the other with an observer. For a detailed discussion see, e.g. Perez Bergliaffa et al. (1996).

Thanks to the leading work of Bell (1964), the ideas of EPR could be tested by experience. Several experiments were made, the latest by Hensen et al. (2015), and the results are clear: QM formalism is not local and incomplete.

According to some authors, the work of EPR and the mentioned experimental results seem to imply that QM is inconsistent with realism. Such statement, however, is based on a questionable hypothesis: locality. A realistic interpretation of QM, only demands to accept non-local effects in entangled systems.

Setting aside locality is polemical, but the reader should notice that not real action at distance between a general class of physical systems is implied. Possible non-local correlations are limited to highly manipulated entangled systems.

In short, QM may manifest non-local effects in order to preserve systemic features with no detriment of ontological realism (see Perez Bergliaffa et al. 1996).

\subsection{Ontology}

Ontological questions in elementary courses on QM are often answered with the so-called wave-particle duality: "depending on the experimental set up, particles may behave as waves, or waves as particles".

This proposition was stated by Louis de Broglie before the formalization of QM was established and was fundamental in a heuristic sense. However, we emphasize that the referents of QM are not particles, nor waves, not even the wave function. The referents of QM are quantum systems, per se.

In other words, quantum systems are not compound by particles nor waves. The latter are classical concepts that do not belong to QM. Classical analogies are heuristically important for the making of a theory, but they have no place in its final postulates (Bunge 1967).

The ontology based on quantum systems may not satisfy our curiosity about the components of the quantum world. In that case, we should proceed to study Quantum Field Theory, a deeper theory that models quantum systems as particular physical fields.

\section{Conclusions}

QM is an extraordinary fundamental physical theory. However, it has been victim of imprecise, subjective, and vague interpretations. In this article we outlined a realistic approach of QM and criticize several misleading propositions that are usually heard around. Inattention on interpretational issues may not affect experimental predictions. However, they engender confusing statements that obscure the theory and hinder further theoretical developments.

From an ethical perspective, vague and confusing statements enhance the action of pseudoscience: QM has been applied to New Age culture, telepathy, pseudo-medicine, the existence of God, mindbody dualism, and other forms of non-sense. All this can be avoided with precise and formal philosophy, essential for interpreting counterintuitive modern physical theories.

\section{References}

Bell, J.S. (1964), “On the Einstein-Podolsky-Rosen Paradox”, Physics 1: 195-200.

Bunge, M.A. (1967), Foundations of Physics, Berlin-Heidelberg-New York: Springer.

Bunge, M.A. (1970), “The So-called Fourth Indeterminacy Relation”, Canadian Journal of Physics 48: 1410.

Bunge, M.A. (1973), Philosophy of Physics, Dordrecht: Reidel.

Bunge, M.A. (1983), Controversias en física, Madrid: Tecnos. 
60 | Federico G. Lopez Armengol y Gustavo E. Romero

Bunge, M.A. (2010), Matter and Mind, Dordrecht-Heidelberg-London-New York: Springer.

Earman, J. (1986), A Primer on Determinism, Dordrecht: Springer.

Einstein, A., Podolsky, B. and N. Rosen (1935), "Can Quantum-Mechanical Description of Physical Reality Be Considered Complete?", Physical Review 47(10): 777-780.

Hensen, B., Bernien, H., Dréau, A.E., Reiserer, A., Kalb, N., Blok, M.S. and W. Amaya (2015), "Loophole-free Bell Inequality Violation Using Electron Spins Separated by 1.3 Kilometres”, Nature 526: 682-686.

Jammer, M. (1974), The Philosophy of Quantum Mechanics: The Interpretations of Quantum Mechanics in Historical Perspective, New York: John Wiley \& Sons, Inc.

Messiah, A. (1981), Quantum Mechanics, Amsterdam: North-Holland.

Perez Bergliaffa, S.E., Vucetich, H. and G.E. Romero (1993), "Axiomatic Foundations of Nonrelativistic Quantum Mechanics: A Realistic Approach”, International Journal of Theoretical Physics 32: 1507-1522.

Perez Bergliaffa, S.E., Romero, G.E. and H. Vucetich (1996), “Axiomatic Foundations of Quantum Mechanics Revisited: The Case for Systems", International Journal of Theoretical Physics 35: 1805-1819.

Popper, K.R. (1959), "The Propensity Interpretation of Probability", The British Journal for the Philosophy of Science 10(37): 25-42.

Schlosshauer, M.A. (2007), Decoherence and the Quantum-to-Classical Transition, Berlin: Springer. 